Research Article

\title{
Research on the Application of an Information System in Monitoring the Dynamic Deformation of a High-Rise Building
}

\author{
Mingzhi Chen $\mathbb{D}^{1},{ }^{1}$ Guojian Zhang $\mathbb{D}^{2,3}$ Chengxin Yu $\mathbb{D}^{1},{ }^{1}$ and Peng Xiao $\mathbb{D}^{1}$ \\ ${ }^{1}$ College of Business, Shandong Jianzhu University, Jinan 250101, China \\ ${ }^{2}$ College of Surveying and Geo-Informatics, Shandong Jianzhu University, Jinan 250101, China \\ ${ }^{3}$ School of Environmental Science and Spatial Informatics, China University of Mining and Technology, Xuzhou 221116, China \\ Correspondence should be addressed to Mingzhi Chen; chenmingzhi1975@126.com, Guojian Zhang; g_j_zhang@cumt.edu.cn, \\ and Chengxin Yu; ycx1108@126.com
}

Received 31 October 2019; Revised 17 April 2020; Accepted 25 June 2020; Published 30 July 2020

Academic Editor: Suzanne M. Shontz

Copyright ( 2020 Mingzhi Chen et al. This is an open access article distributed under the Creative Commons Attribution License, which permits unrestricted use, distribution, and reproduction in any medium, provided the original work is properly cited.

\begin{abstract}
With the acceleration of urbanization, there are increasingly more high-rise buildings in cities. In turn, high-rise building collapse accidents occur frequently. The causes of the danger, in addition to the extremely severe stress, are predominantly due to the longterm role of unstable factors, resulting in an unrecoverable internal structure. Therefore, it is advisable to monitor the dynamic deformation of buildings to prevent accidents. However, there is no particularly mature application system on the market to enable this monitoring, so our research group has carried out a long-term research in this field. This paper introduces the design of a set of practical information systems by using information technology and the principle of close-range photogrammetry. The system used the photographing scale transformation-time baseline parallax (PST-TBP) method to analyse image data collected with a digital camera, implement close-range photogrammetry, and input the image data into a computer. A building deformation diagram is obtained using our own software. The associated deformation curve can clearly reproduce the building deformation trend to monitor the building health. We conducted many laboratory simulation experiments to verify the information system, and the verification results prove that this process is rigorous. To apply this information system to a real-life scenario as soon as possible, we further studied its application to high-rise buildings, improved the system by using data and experience obtained by monitoring the tallest local building, and achieved good results. Finally, combined with the development of current intelligent technology, directions for system improvement are explored.
\end{abstract}

\section{Introduction}

The process of urbanization is currently accelerating, city areas are expanding, and the population has increased rapidly. In addition, there are increasingly more high-rise buildings. However, the collapse of high-rise buildings is also frequent, and the hidden danger of these hazards has received increasingly more attention [1-3]. The causes of the danger, in addition to the extremely severe stress, are predominantly due to the long-term role of unstable factors, resulting in an unrecoverable internal structure, but the associated microdeformation processes are difficult to monitor $[4,5]$. If we can obtain real-time deformation data from these buildings, then these data can be superimposed and compared, and we can clearly analyse whether the building is in a state of health. If the deformation is excessive or unrecoverable in an unhealthy state, collapse can be prevented in advance, which is a very effective method to avoid accidents [6-9].

The study of building deformation monitoring to ensure its safety has been started since the 1990s. In 2000, Luo et al.'s and other experiments verified that GNSS (Global Navigation Satellite System) could be used to monitor the lowfrequency dynamic characteristics of high-rise buildings [10]. In 2006, Lee and others used digital cameras to monitor displacement and compared the results with those of a laser vibrator [11]. Chen Weihuan and others used a digital camera to monitor the dynamic displacement of Guangzhou new TV tower in real time in 2011, and compared with the dynamic displacement data measured by GNSS, it is 
basically consistent. In 2012, Kuang et al. used GNSS to monitor the dynamic response of high-rise buildings under typhoon loads and to monitor the dynamics of a high-rise building under construction in Hong Kong [12]. In 2018, a smart SHM system with 46 sensors at Shanghai World Financial Center (SWFC) in China is validated for the analysis of dynamic characteristic of high building by earthquake. And at the same time, Zhou utilized a tomography-based persistent scatterers interferometry monitoring the deformation performances of high-rise building, i.e., SWFC and JinMao Tower, in Shanghai Lujiazui Zone. The above scholars have done a lot of work in the field of high-level health monitoring, our scheme is different from them, and our monitoring of real-time instantaneous dynamic deformation has new research.

After years of research, our research group designed a set of information systems to combine information technology with digital photogrammetry. Digital photography [13-15] can observe the structural deformation of buildings, and the combination of digital photography and information technology can make this technology faster and more efficient than the alternatives. Then, we verified the scientific foundation of this method by simulating and making various building models in the laboratory. Several basic experiments can prove that the method is efficient and scientific. In addition, we collected and monitored the instantaneous dynamic deformation image of buildings and draw the associated graph. To further apply the systems in practice, we need to study their practical application in a wide variety of buildings; our aim is thus to study the application of deformation monitoring information systems in high-rise buildings.

This paper briefly introduces the principle and workflow of one of the information systems and then describes a typical simulation experiment for scientific verification. It also focuses on how we can monitor the first tall building in Jinan, China and obtain first-hand data and experience of field monitoring and explains how to further improve the function of the information system in the process of overcoming various problems in real time and incorporate recent advancements in intelligent technology at the present stage. Finally, new improvements and development directions are put forward.

\section{Construction of the Dynamic Deformation Monitoring Information System}

2.1. Accuracy Assessment of Test Camera. The distortion of the camera in the photographic system plays a major role in influencing its measurement accuracy [16-20]. Considering that the distortion error is linear near the central position of the images [21], we adopted a grid method [22] in the study to eliminate the distortion of the digital camera to improve the measurement accuracy. Figure 1 illustrates that the distortion error of the deformation point on the object moves from Position $\mathrm{A}\left(x_{\mathrm{A}}, z_{\mathrm{A}}\right)$ to Position $\mathrm{A}^{\prime}\left(x_{\mathrm{A}^{\prime}}, z_{\mathrm{A}^{\prime}}\right)$ in the camera's view and that $\Delta X_{\mathrm{AA}^{\prime}}$ and $\Delta Z_{\mathrm{AA}^{\prime}}$ are the corresponding horizontal and vertical displacements of this deformation point, respectively.

After modifying the distortion of test camera, we used the direct linear transformation (DLT) method [23] to assess

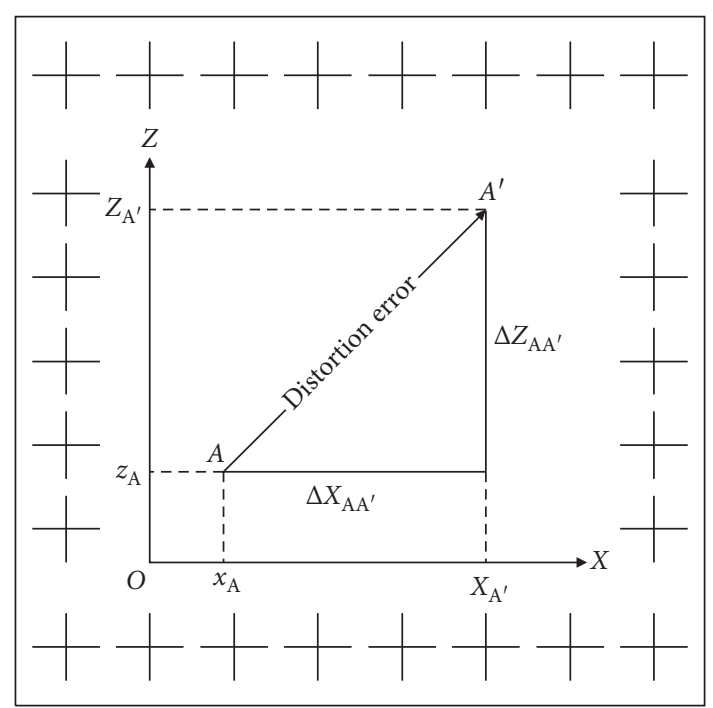

FIGURE 1: Influence of distortion error.

its measurement accuracy. The DLT method is shown as follows:

$$
\left.\begin{array}{l}
x-\frac{L_{1} X+L_{2} Y+L_{3} Z+L_{4}}{L_{9} X+L_{10} Y+L_{11} Z+1}=0 \\
z-\frac{L_{5} X+L_{6} Y+L_{7} Z+L_{8}}{L_{9} X+L_{10} Y+L_{11} Z+1}=0
\end{array}\right\}
$$

where $(x, z)$ are the image plane coordinates of deformation points without errors, $(X, Y, Z)$ are the space coordinates of the corresponding deformation points, and $L_{i}(i=1,2,3, \ldots, 11)$ are the functions of the exterior and interior parameters of test camera.

The spatial coordinates of the reference points and deformation points were measured by a total station in the laboratory. The DLT method was used to calculate the spatial coordinates of deformation points De 0 and De 1 based on the coordinates in Tables 1 and 2. Their differences were obtained by comparing the actual coordinates of De 0 and De 1 with their calculated coordinates (Table 3 ). The maximal and minimal measurement errors were $2 \mathrm{~mm}$ and $0 \mathrm{~mm}$, respectively, with an average measurement error of $1 \mathrm{~mm}$.

2.2. Monitoring Principle. The monitoring principle of the dynamic deformation monitoring information system (DDMIS) is time baseline parallax based on photographing scale transformation (PST-TBP) method. The PST-TBP method is detailed in [24-27] and Figure 2 shows its schematic diagram. Part of the PST-TBP method is as follows.

In Figure 2(b), on the object plane, $\Delta x^{\text {def }}$ and $\Delta z^{\text {def }}$ of the corresponding deformation point are

$$
\left.\begin{array}{l}
\Delta x^{\mathrm{def}}=\frac{\mathrm{SA}}{\mathrm{Sa}} \Delta p_{x}^{\mathrm{def}}=m \cdot \Delta p_{x}^{\mathrm{def}}, \\
\Delta z^{\mathrm{def}}=\frac{\mathrm{SA}}{\mathrm{Sa}} \Delta p_{z}^{\mathrm{def}}=m \cdot \Delta p_{z}^{\mathrm{def}},
\end{array}\right\}
$$


TABLE 1: Spatial coordinates of reference points R0 to R7 (m).

\begin{tabular}{lcccccccc}
\hline Point number & R0 & R1 & R2 & R3 & R4 & R5 & R6 \\
\hline$X$ & 108.343 & 109.739 & 110.144 & 110.144 & 109.492 & 108.862 & 108.456 & 108.713 \\
$Y$ & 95.770 & 95.804 & 95.957 & 95.957 & 97.022 & 97.199 & 96.194 & 96.487 \\
$Z$ & 99.534 & 99.542 & 99.540 & 99.540 & 99.568 & 99.240 & 99.225 & 99.384 \\
\hline
\end{tabular}

TAble 2: Pixel coordinates of reference points (R0 to R7) and deformation points (De0 and De 1) (pixel).

\begin{tabular}{cccccccccccc}
\hline \multicolumn{2}{c}{ Point number } & R0 & R1 & R2 & R3 & R4 & R5 & R6 & R7 & De0 & De1 \\
\hline \multirow{2}{*}{ Photo 1} & $X$ & 205 & 428 & 581 & 958 & 1540 & 1864 & 782 & 1120 & 429 & 1541 \\
& $Z$ & 687 & 468 & 437 & 451 & 490 & 924 & 1057 & 796 & 700 & 718 \\
\hline \multirow{2}{*}{ Photo 2} & $X$ & 144 & 1179 & 1486 & 1716 & 1918 & 1631 & 487 & 907 & 619 \\
& $Z$ & 572 & 495 & 491 & 530 & 605 & 1101 & 948 & 799 & 643 & 826 \\
\hline
\end{tabular}

TABle 3: Accuracy assessment for deformation points De0 and De1.

\begin{tabular}{lccc}
\hline Name & Actual coordinates $(\mathrm{m})$ & Calculated coordinates $(\mathrm{m})$ & Differences $(\mathrm{mm})$ \\
\hline De0- $X$ & 108.825 & 108.826 & 1 \\
De0-Y & 95.887 & 95.888 & 1 \\
De0- $Z$ & 99.441 & 99.440 & 1 \\
De1-X & 109.067 & 109.065 & 2 \\
De1- $Y$ & 96.935 & 96.934 & 1 \\
De1- $Z$ & 99.394 & 99.394 & 0 \\
\hline
\end{tabular}

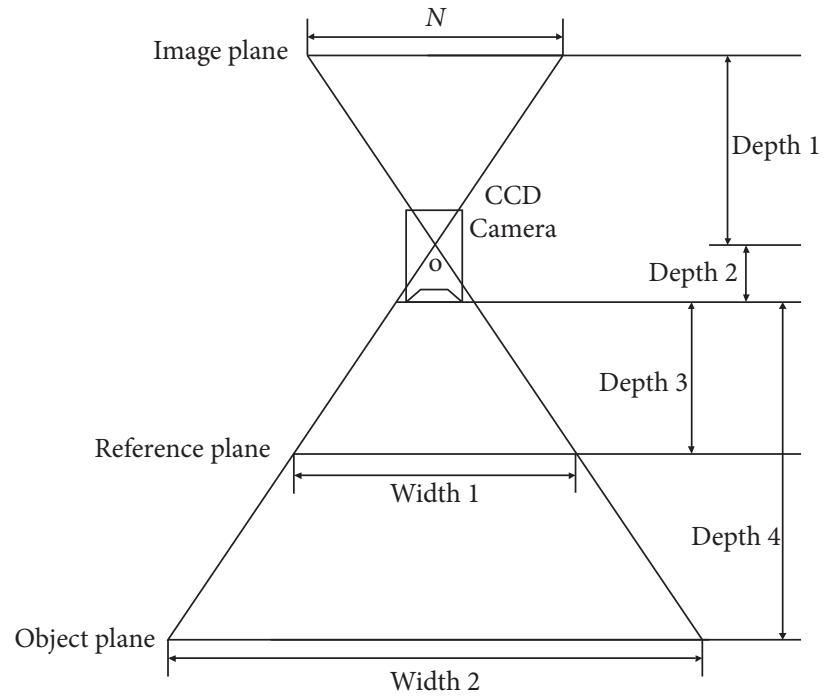

(a)

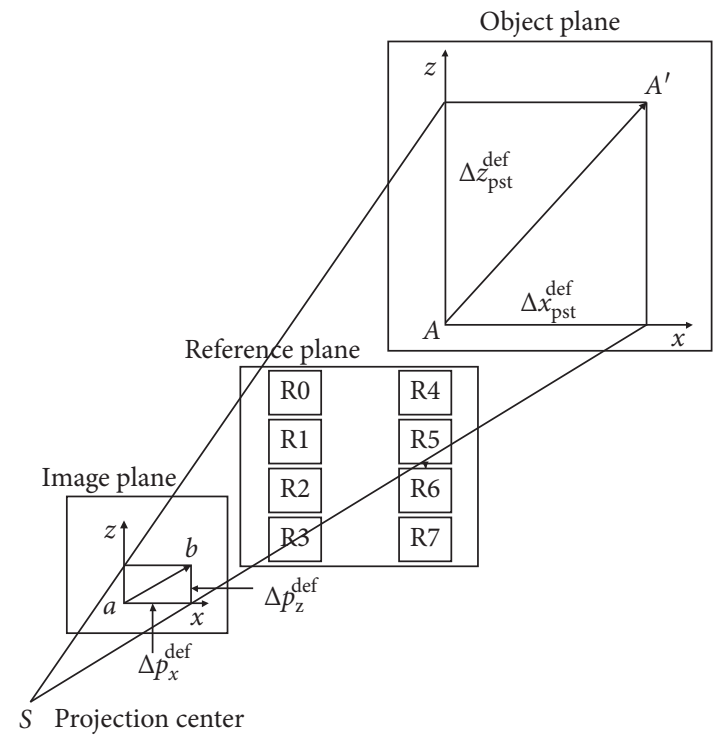

(b)

Figure 2: Photographing scale transformation-time baseline parallax method [24-27].

where $m$ is the photographing scale on the reference plane, $\Delta x^{\mathrm{def}}$ and $\Delta x^{\mathrm{def}}$ are the horizontal and vertical deformation of deformation point on the object plane, and $\Delta p_{x}^{\text {def }}$ and $\Delta p_{z}^{\text {def }}$ are the horizontal and vertical parallax of the correspondence image point on the image plane. Note that there exist systematic errors in $\Delta p_{x}^{\text {def }}$ and $\Delta p_{z}^{\text {def }}$.

Then, the corrected parallax of the corresponding deformation points is obtained:

$$
\left.\begin{array}{l}
\operatorname{cor} \Delta p_{x}^{\text {def }^{\prime}}=\Delta p_{x}^{\text {def }}-\Delta p_{x}^{\text {def0' }}, \\
\operatorname{cor} \Delta p_{z}^{\text {def }^{\prime}}=\Delta p_{z}^{\text {def }}-\Delta p_{z}^{\text {def0' }},
\end{array}\right\}
$$

where $\left(\operatorname{cor} \Delta p_{x}^{\text {deff }}, \operatorname{cor} \Delta p_{z}^{\text {deff }}\right)$ are the corrected parallax of deformation points in the coordinate system after the barycentralization. $\left(\Delta p_{x}^{\text {def } b}, \Delta p_{z}^{\text {def }}\right)$ are the systematic error of the deformation point in the coordinate system after the barycentralization, respectively.

Then, we obtain the corrected displacements of deformation points based on the control plane:

$$
\left.\begin{array}{l}
\operatorname{cor} \Delta x^{\mathrm{def}}=m \cdot \operatorname{cor} \Delta p_{x}^{\mathrm{def}^{\prime}}, \\
\operatorname{cor} \Delta z^{\mathrm{def}}=m \cdot \operatorname{cor} \Delta p_{z}^{\mathrm{def}^{\prime}},
\end{array}\right\}
$$


where ( $\operatorname{cor} \Delta x^{\text {def }}, \operatorname{cor} \Delta z^{\text {def }}$ ) are the corrected displacements on the reference plane of deformation points.

According to the photographing scale difference between the reference plane and the object plane, we got the actual deformation of the deformation points:

$$
\left.\begin{array}{l}
\Delta x_{\mathrm{pst}}^{\mathrm{def}} i=\Delta \mathrm{pstc} \cdot \operatorname{cor} \Delta x^{\mathrm{def}}, \\
\Delta z_{\mathrm{pst}}^{\mathrm{def}} i=\Delta \mathrm{pstc} \cdot \operatorname{cor} \Delta z^{\mathrm{def}},
\end{array}\right\}
$$

where $\Delta x_{\mathrm{pst}}^{\mathrm{def}} i$ and $\Delta z_{\mathrm{pst}}^{\mathrm{def}} i$ are the actual spatial deformations on the object plane of deformation, $i=1,2,3, \ldots, n, \Delta \mathrm{pstc}$ is the coefficient of the photographing scale transformation, and $\Delta$ pstc $=$ Depth4/Depth3.

\subsection{Accuracy Assessment of DDMIS in the Laboratory.} Before test on the high building, the reliability of DDMIS should be investigated in the laboratory. We used a Sony-350 camera in this study to monitor the instantaneous deformation of a steel frame when it was impacted by a dumbbell. Table 4 shows the parameters of the Sony-350 camera. Figure 3(b) shows the test site. Points labeled as U0-U4 are deformation points. Points labeled as $\mathrm{C} 0-\mathrm{C} 9$ are reference points, which formed the reference plane. The test process and impact loads are detailed in [28].

After processing the images, we obtained the measurement accuracy of DDMIS. Table 5 shows that the average absolute accuracy and relative accuracy were $0.28 \mathrm{~mm}$ and $1.1 \%$, respectively. This suggests that DDMIS in this study can meet the accuracy requirements of high-rise buildings monitoring.

\section{Field Experiment}

This information system has achieved very good results through the simulation experiments of typical structures, but if we apply it to practice, we still have to overcome many difficulties. For example, the structure of the building itself, the photography scene environment, the changing weather during the image capture process, and the people and vehicles around the building should be investigated to further complete the DDMIS. Thus, this paper tried to use DDMIS to monitor high building.

3.1. Experiment Site Selection. We chose the main building of Hanyu Jingu, a tall building in Jinan, which is 339 meters high. It is also surrounded by other tall buildings, so it is impossible to set up cameras at close range to capture deformed images at the top of the tallest building.

After several days of peripheral research and coordination, the final image capture location was selected to be a nearby hill less than 200 meters above sea level; there is a manmade cement flat floor in front of the main building of Hanyu Jingu that is suitable for arranging cameras and reference signs.

\subsection{Optimization and Design of the Field Schematic Diagram.} Since the monitoring distance for this building is longer than that for the laboratory, this distance can fully meet the accuracy requirements for high-definition digital cameras at this stage. However, the arrangement of reference points and the selection of deformation points are difficult. To solve this problem, we improved the experimental principle. We use the image matching-time baseline parallax method to form a reference plane perpendicular to the photography direction by setting a stable reference point not far from the camera, as shown in Figure 4. In the data calculation, the follow-up image is matched with the reference picture, and then the parallax caused by external factors is eliminated.

3.3. Improvement of the Information System Workflow. Before the data collection, we also improved the workflow of the information system, integrating the field investigation and the preplan setting into the operation process of the information system. The improved scheme is as follows:

(1) Site investigation: conduct an investigation of the building site, and then determine the basic information of the building structure, age, load, local terrain, and so on.

(2) Monitoring point selection: according to the tall building structure and structural mechanics, in addition to other principles, confirm the important and requested monitoring points.

(3) Camera arrangement and deviation correction: we arrange digital cameras in multiple directions and reconcile them before use in order to eliminate the effect of distortion difference, which solves the problem that the digital cameras have no internal or external coordinates.

(4) Data acquisition: we use several digital cameras to collect high-resolution multiangle and omnidirectional building deformation images. We collect a large amount of photographical data to obtain a normal distribution confidence interval.

(5) Computer software analysis: the advantage of digital cameras is that they can transmit digital signals; the photographs we take are transferred to the computer in the form of a digital signal. When analysing a large amount of data with our self-developed computer imaging analysis software, the main job is the content processing and deformation value calculation.

(6) Content processing includes bitmap format conversion, reading reference point pixel position data, reading deformation point pixel position data, calculating the number of reference points, calculating the number of deformation points, and calculating the photograph scale coefficient.

(7) The step of calculating the deformation value is as follows: coordinate centralization, centering the reference point pixel coordinates; calculating the correction coefficients of reference point inspection; finding the centralization coordinates of deformation points; finding the positive value of the parallax change of the deformation point; and finding the 
TABle 4: Parameters of Sony-350 camera [28].

\begin{tabular}{lcccc}
\hline Type & Sensor & Sensor scale & Focal length & Active pixels \\
\hline Sony DSLR A350 (Sony-350) & CCD & $23.5 \times 15.7 \mathrm{~mm}$ & $35 \mathrm{~mm}(27-375)$ & $4592 \times 3056$ pixels \\
\hline
\end{tabular}

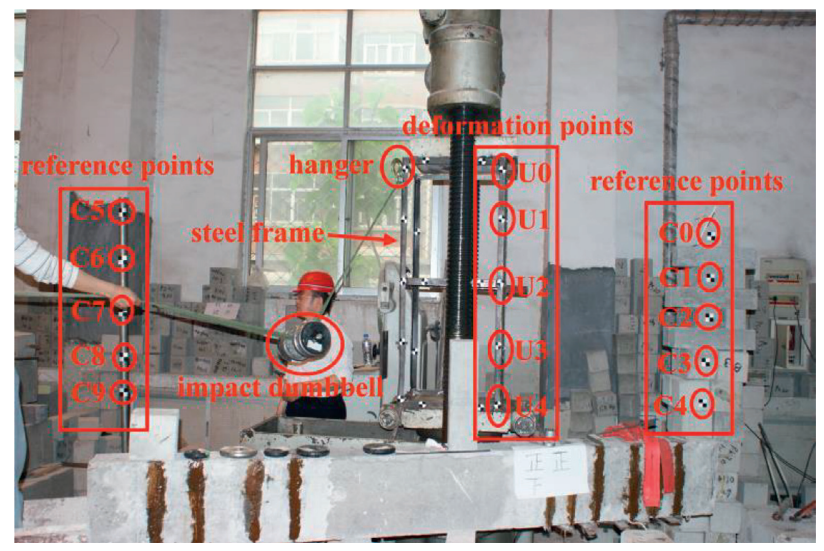

Figure 3: Impact test on a steel frame [28].

TABle 5: Measurement accuracy [28].

\begin{tabular}{lcccc}
\hline Line & Actual length $(\mathrm{mm})$ & Measured length $(\mathrm{mm})$ & Absolute accuracy $(\mathrm{mm})$ & Relative accuracy $(\%)$ \\
\hline U2-U3 & 296.80 & 296.90 & 0.10 & 0.34 \\
U3-U4 & 278.80 & 278.60 & 0.20 & 0.72 \\
C2-C3 & 241.40 & 241.20 & 0.20 & 0.83 \\
C6-C7 & 253.90 & 253.30 & 0.60 & 2.40 \\
C7-C8 & 225.10 & 225.40 & 0.30 & 1.30 \\
\multicolumn{2}{r}{ Average } & & 0.28 & 1.10 \\
\hline
\end{tabular}

apparent difference after correction of the deformation point.

(8) An analysis report of the monitoring results is issued on site. The entire solution process is completed in as fast as 15 minutes. The integration of internal and external operations is realized, and the efficiency and accuracy of monitoring are greatly improved over previous efforts.

In this paper, we use two cameras to obtain the deformation data. Then, we can verify the accuracy of DDMIS.

According to the improved schematic diagram, we set up the reference points $\mathrm{C} 0, \mathrm{C} 1, \mathrm{C} 2$, and $\mathrm{C} 3$, as shown in Figures 4(a) and 4(b). Because the tall building is very high, we cannot put our own design marks for deformation points on it, so we also improved the workflow of the information system. First, we take pictures of tall buildings and then look for marks from the building itself. These marks locations are as follows in pictures:

(1) They have an exact intersection.

(2) The point position has a high computer resolution. As Figures 4(a) and 4(b) show, the points we are looking for are $\mathrm{U} 0, \mathrm{U} 1$, and $\mathrm{U} 2$.

\section{Data Analysis}

Through data processing, the measurement accuracy of Cameras 1 and 2 (Tables 6 and 7) and deformation values of the deformation points (Tables 8 and 9) were obtained. In the test, the pixel displacements of the reference points were supposed to be zero in theory. However, their pixel displacements were not zero in DDMIS. As such, these values were deemed as the measurement accuracy of DDMIS. Table 6 shows the average measurement accuracy of Camera 1. Table 7 shows the average measurement accuracy of Camera 2. Moreover, Table 7 shows that the average displacements of $\mathrm{C} 0-\mathrm{C} 3$ were 1.17 pixels, 0.74 pixels, 0.59 pixels, and 0.28 pixels, respectively. Table 7 shows that the average displacements of $\mathrm{C} 0-\mathrm{C} 3$ were 1.16 pixels, 0.99 pixels, 0.34 pixels, and 0.54 pixels, respectively. The measurement accuracy of DDMIS reached subpixel in the field experiment. Thus, deformation values of the deformation points in Tables 8 and 9 were reliable.

In order to assess the high-rise buildings health situation on the test field, the deformation curves of the high building (Figures 4-8) were obtained in real time by DDMIS and they are useful to study the high-rise buildings dynamic properties. 


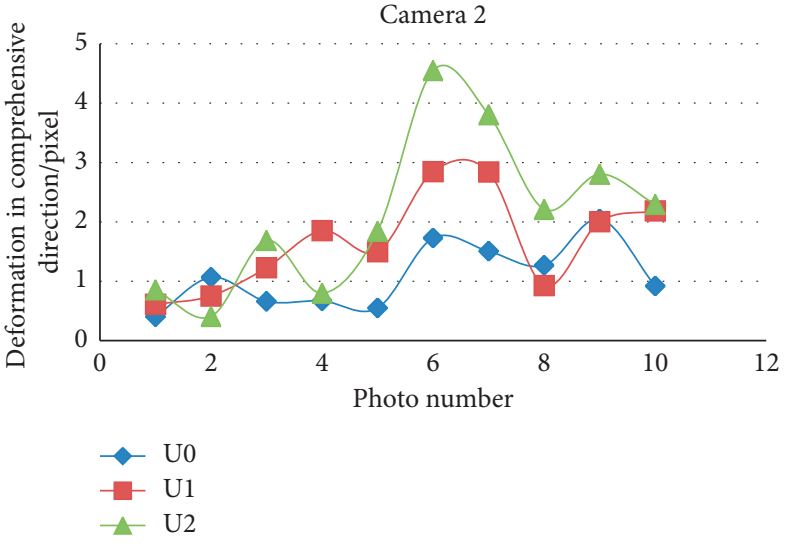

(a)

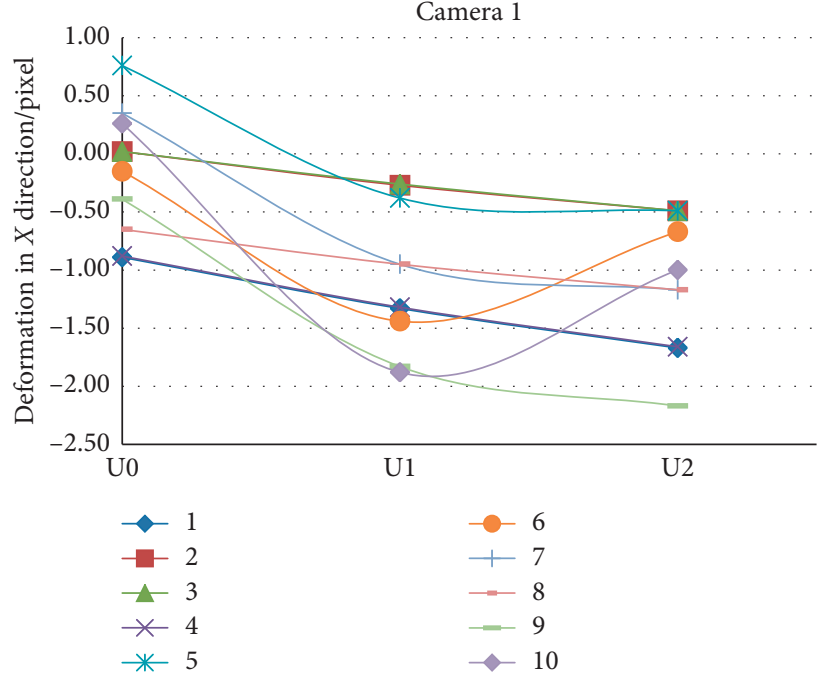

(b)

Figure 4: (a) Deformation curves of deformation points in comprehensive direction from Camera 2. (b) High-building global deflection curves from Camera 1.

TABle 6: Measurement accuracy of Camera 1 (pixel).

\begin{tabular}{lccccccc}
\hline & C0 & & \multicolumn{2}{c}{ C1 } & \multicolumn{2}{c}{ C2 } & \multicolumn{2}{c}{ C3 } \\
\hline$X$ & $Z$ & $X$ & $Z$ & $X$ & $Z$ & $X$ & $Z$ \\
0.80 & 0.85 & 0.65 & 0.37 & 0.22 & 0.55 & 0.06 & 0.27 \\
\multicolumn{2}{c}{1.17} & \multicolumn{2}{c}{0.74} & \multicolumn{2}{c}{0.59} & \multicolumn{2}{c}{0.28} \\
\hline
\end{tabular}

TABLE 7: Measurement accuracy of Camera 2 (pixel).

\begin{tabular}{lcccccccc}
\hline & $\mathrm{C} 0$ & & \multicolumn{2}{c}{$\mathrm{C} 1$} & & $\mathrm{C} 2$ & \multicolumn{2}{c}{$\mathrm{C} 3$} \\
\hline$X$ & $Z$ & $X$ & $Z$ & $X$ & $Z$ & $X$ & $Z$ \\
0.63 & 0.98 & 0.21 & 0.97 & 0.15 & 0.30 & 0.49 & 0.22 \\
\multicolumn{2}{c}{1.16} & \multicolumn{2}{c}{0.99} & \multicolumn{2}{c}{0.34} & \multicolumn{2}{c}{0.54} \\
\hline
\end{tabular}

TABle 8: Deformation data obtained with Camera 1.

\begin{tabular}{lcccccc}
\hline Photo number & DX0 & DZ0 & DX1 & DZ1 & DX2 & DZ2 \\
\hline 1 & -0.89 & -0.65 & -1.33 & -0.95 & -1.67 & -2.17 \\
2 & 0.02 & 0.76 & -0.27 & 0.62 & -0.49 & -0.49 \\
3 & 0.02 & 0.26 & -0.26 & 1.12 & -0.49 & 0.00 \\
4 & -0.88 & 0.00 & -1.32 & 1.00 & -1.66 & 0.00 \\
5 & 0.76 & -0.65 & -0.38 & -0.95 & -0.49 & -0.17 \\
6 & -0.15 & 0.26 & -1.44 & 0.12 & -0.67 & 0.00 \\
7 & 0.35 & -0.74 & -0.95 & -0.88 & -1.17 & -1.00 \\
8 & -0.65 & 0.26 & -0.95 & 0.12 & -1.17 & 0.01 \\
9 & -0.39 & 0.26 & -1.83 & 0.12 & -2.17 & -0.99 \\
10 & 0.26 & 0.67 & -1.88 & -0.32 & -1.00 & -0.31 \\
\hline
\end{tabular}

In Figure 6, deformation points U0, U1, and U2 are in elastic deformation in $X$ direction. The maximum deformation values of deformation points $\mathrm{U} 0, \mathrm{U} 1$, and $\mathrm{U} 2$ are 0.89 pixels (on Photo 5), 1.88 pixels (on Photo 10), and 2.17 pixels (on Photo 9), respectively. As we all know, the high-rise buildings are always shaking, and it shook violently with the increasing height along high-rise buildings. Thus, in $X$
TABLe 9: Deformation data obtained with Camera 2.

\begin{tabular}{lcccccc}
\hline Photo number & DX0 & DZ0 & DX1 & DZ1 & DX2 & DZ2 \\
\hline 1 & -0.38 & -0.13 & -0.38 & 0.48 & 0.62 & -0.59 \\
2 & -0.86 & -0.63 & -0.72 & 0.22 & 0.39 & 0.12 \\
3 & 0.62 & -0.23 & 0.62 & -1.06 & 1.63 & -0.44 \\
4 & -0.23 & 0.63 & 0.78 & 1.68 & 0.78 & 0.18 \\
5 & -0.23 & -0.50 & 0.78 & 1.28 & 1.79 & -0.44 \\
6 & 1.41 & 1.00 & 2.70 & 0.90 & 3.92 & 2.31 \\
7 & 0.28 & 1.48 & 1.29 & 2.53 & 2.29 & 3.04 \\
8 & 0.78 & 1.00 & -0.23 & 0.90 & 1.79 & 1.30 \\
9 & 1.79 & 1.00 & 1.79 & 0.90 & 2.79 & 0.29 \\
10 & 0.92 & -0.01 & 1.06 & 1.91 & 2.18 & -0.72 \\
\hline
\end{tabular}

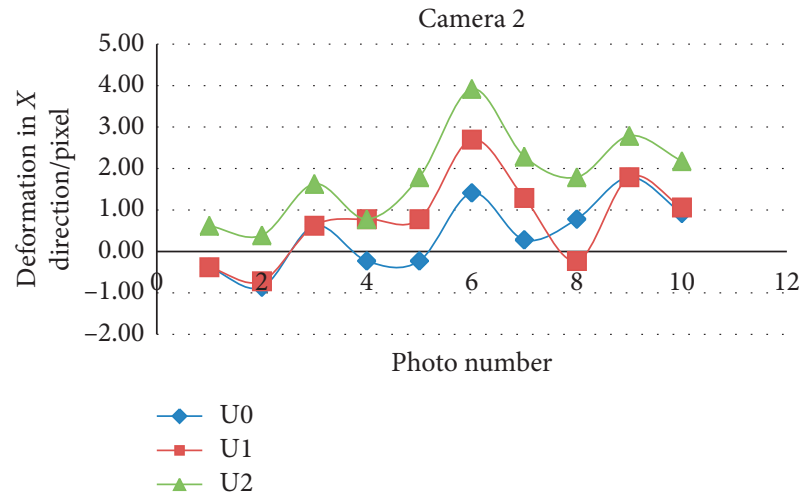

FIgURE 5: Deformation curves of deformation points in $X$ direction from Camera 2.

direction, the monitoring results are consistent with the vibration rule of high-rise buildings.

In Figure 5, deformation points U0, U1, and U2 are in elastic deformation in $X$ direction. The maximum 


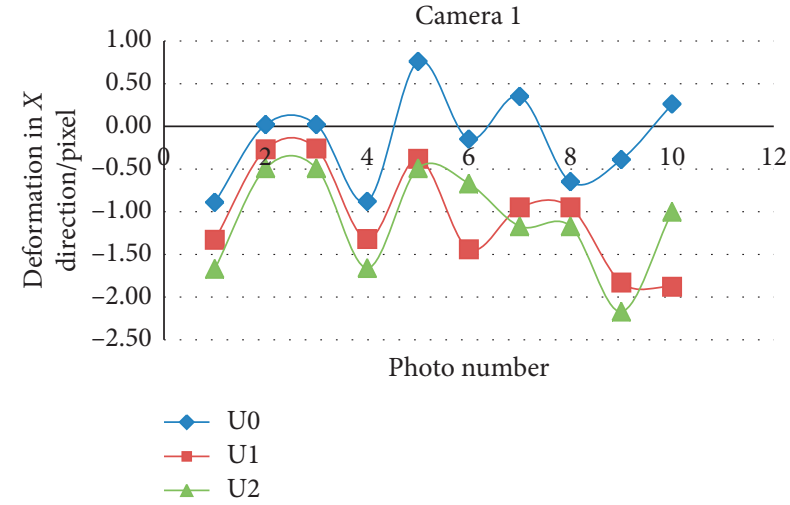

Figure 6: Deformation curves of deformation points in $X$ direction from Camera 1.

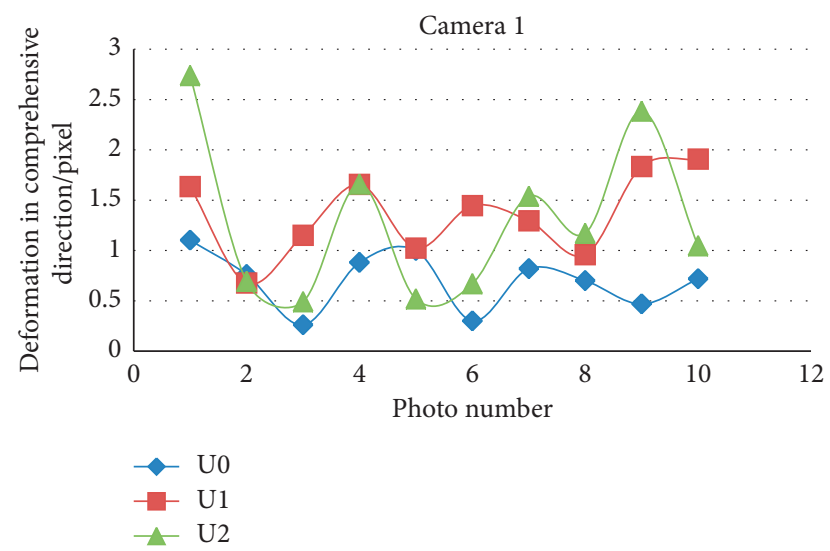

Figure 7: Deformation curves of deformation points in comprehensive direction from Camera 1.

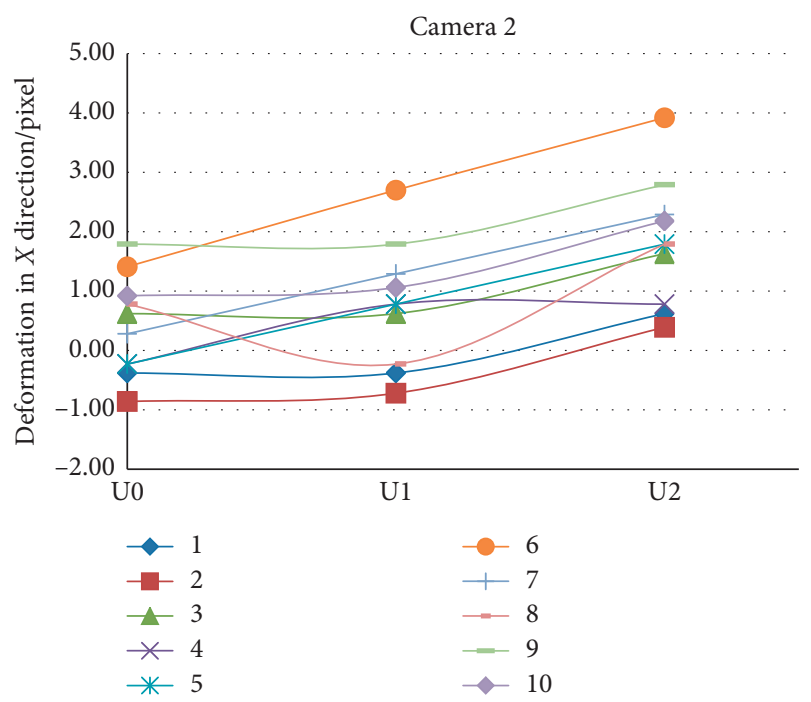

FIgURE 8: High-building global deflection curves from Camera 2.

deformation values of deformation points $\mathrm{U} 0, \mathrm{U} 1$, and $\mathrm{U} 2$ are 1.79 pixels (on Photo 6), 2.70 pixels (on Photo 6), and 3.92 pixels (on Photo 6), respectively. Thus, in $X$ direction, the monitoring results are also consistent with the vibration rule of high-rise buildings.

In Figure 7, deformation points U0, U1, and U2 are in elastic deformation in comprehensive direction. The maximum deformation values of deformation points $\mathrm{U} 0$, $\mathrm{U} 1$, and $\mathrm{U} 2$, are 1.10 pixels (on Photo 1), 1.91 pixels (on Photo 10), and 2.74 pixels (on Photo 1), respectively. Thus, in comprehensive direction, the monitoring results are also consistent with the vibration rule of high-rise buildings.

In Figure 4(a), deformation points U0, U1, and U2 are in elastic deformation in comprehensive direction. The maximum deformation values of deformation points U0, U1, and U2 are 2.05 pixels (on Photo 6), 2.84 pixels (on Photo 6), and 4.55 pixels (on Photo 6), respectively. Thus, in comprehensive direction, the monitoring results are also consistent with the vibration rule of high-rise buildings.

In Figure 4(b), the global deformation of Hanyu Jin'gu high-rise buildings is in elastic deformation in $X$ direction. From Photo 1 to Photo 6, the shape of high-rise buildings changed from line to left semiparabola and parabola. From Photo 7 to Photo 10, the shape of high-rise buildings changed from left semiparabola to line and parabola. It is very different from the vibration rule of a flexible cantilever beam.

In Figure 8, the global deformation of Hanyu Jin'gu high-rise buildings is in elastic deformation in $X$ direction. From Photo 1 to Photo 6, the shape of high-rise buildings changed from line to right semiparabola and line. From Photo 7 to Photo 10, the shape of high-rise buildings changed from line to parabola and right semiparabola. It is also very different from the vibration rule of a flexible cantilever beam.

According to the monitoring results, we found that the global deformation of Hanyu Jin'gu high-rise buildings is complex. Their dynamic properties are completely different from that of a low-rise building. This paper also provides technical support for mastering the dynamic characteristics of high-rise buildings and their real-time security early warning.

Note that it is impossible to monitor the high-rise buildings with high accuracy since the measurement distance is as far as $700 \mathrm{~m}$, and digital camera pixels are limited. And we used two cameras to monitor Hanyu Jin'gu high-rise buildings for making the monitoring results reliable.

\section{Conclusions}

This study uses monocular digital photography based on the photographing scale transformation-time baseline parallax (PST-TBP) method, to monitor the instantaneous dynamic global deformation of a high-rise building in natural state. Two digital cameras were used to monitor the high-rise buildings to support each other. Deformation curves of the high-rise buildings were depicted by the dynamic deformation monitoring information system (DDMIS) to study the dynamic properties of the high-rise buildings. Through processing the image sequences of the high-rise buildings, the following conclusions are obtained: 
(1) The measurement accuracy of DDMIS reached subpixel in $X$ and $Z$ directions. From Camera 1, the average displacements of $\mathrm{C} 0-\mathrm{C} 3$ in $X$ and $Z$ directions were 0.80 and 0.85 pixels, 0.65 and 0.37 pixels, 0.22 and 0.55 pixels, and 0.06 and 0.27 pixels, respectively. From Camera 2, the average displacements of $\mathrm{C} 0-\mathrm{C} 3$ were 0.63 and 0.98 pixels, 0.21 and 0.97 pixels, 0.15 and 0.30 pixels, and 0.49 and 0.22 pixels, respectively.

(2) Deformation points on the high-rise buildings are in elastic deformation in $X$ and comprehensive direction. The high-rise buildings are always shaking, and it shook violently with the increasing height along highrise buildings. The maximum deformation values of deformation point $\mathrm{U} 2$ in $X$ and comprehensive direction are 3.92 and 4.55 pixels, respectively.

(3) The global deformation of Hanyu Jin'gu high-rise buildings is complex, and their dynamic properties are completely different from that of a low-rise building. In natural state, the shape of Hanyu Jin'gu high-rise buildings changed back and forth from oblique line to semiparabola and parabola.

In conclusion, the Hanyu Jin'gu high-rise buildings were in good health at the time of testing. This study proves that DDMIS can depict the deformation trend curves of the highrise buildings which are useful for studying the high-rise buildings dynamic properties. Moreover, the deformation trend curves of the high-rise buildings also can be used to assess the high-rise buildings health situation and warn the possible danger of the high-rise buildings. This information is essential for making decisions regarding the high-rise buildings health. This experiment also provides valuable experience for the on-site analysis of more types of buildings, and it also prompts the research group to further improve the safety deformation monitoring information system.

With the advent of the era of computer intelligence, many intelligent devices have appeared. For example, the clarity of digital cameras is improving, and it is also possible to connect to a wireless network to transmit the image capture information to a computer system in real time. The realization of this technology is conducive to real-time monitoring of the health status of buildings. At present, the functions of various intelligent handheld devices are becoming increasingly mature. A high-end smartphone or palmtop tablet already has its own image capture function comparable to a professional digital camera, and these smart devices themselves can run image processing software at high speed. For using such an intelligent device in the future, we recommend incorporating our building safety information system into application software running on smart devices to simplify the operation of this system. This technology can be applied to the health monitoring of various buildings at any time or place and provides a powerful guarantee for human health and safety.

\section{Data Availability}

The data used to support the findings of this study are available from the corresponding author upon request.

\section{Disclosure}

Chengxin $\mathrm{Yu}$, Mingzhi Chen, and Guojian Zhang are the first, second, and third corresponding authors, respectively.

\section{Conflicts of Interest}

The authors declare that there are no conflicts of interest regarding the publication of this paper.

\section{Acknowledgments}

The authors gratefully acknowledge the financial support from the Science and Technology Project of Shandong Province of China (Grant no. 2010GZX20125).

\section{References}

[1] T.-H. Yi, H.-N. Li, and M. Gu, "Recent research and applications of GPS-based monitoring technology for high-rise structures," Structural Control and Health Monitoring, vol. 20, no. 5, pp. 649-670, 2013.

[2] T.-H. Yi, H.-N. Li, and M. Gu, "Optimal sensor placement for health monitoring of high-rise structure based on genetic algorithm," Mathematical Problems in Engineering, vol. 2011, Article ID 395101, 12 pages, 2011.

[3] T.-H. Yi, H.-N. Li, and M. Gu, "A new method for optimal selection of sensor location on a high-rise building using simplified finite element model," Structural Engineering and Mechanics, vol. 37, no. 6, pp. 671-684, 2011.

[4] Z. Lu, D. Wang, S. F. Masri, and X. Lu, "An experimental study of vibration control of wind-excited high-rise buildings using particle tuned mass dampers," Smart Structures and Systems, vol. 18, no. 1, pp. 93-115, 2016.

[5] M. Kuwabara, S. Yoshitomi, and I. Takewaki, "A new approach to system identification and damage detection of highrise buildings," Structural Control and Health Monitoring, vol. 20, no. 5, pp. 703-727, 2013.

[6] J.-W. Park, J.-J. Lee, H.-J. Jung, and H. Myung, "Vision-based displacement measurement method for high-rise building structures using partitioning approach," NDT \& E International, vol. 43, no. 7, pp. 642-647, 2010.

[7] M. H. Rafiei and H. Adeli, "A novel machine learning-based algorithm to detect damage in high-rise building structures," The Structural Design of Tall and Special Buildings, vol. 26, pp. 1-11, 2017.

[8] I. Venanzi, F. Ubertini, and A. L. Materazzi, "Optimal design of an array of active tuned mass dampers for wind-exposed high-rise buildings," Structural Control and Health Monitoring, vol. 20, no. 6, pp. 903-917, 2013.

[9] J.-H. Lee, H.-N. Ho, M. Shinozuka, and J.-J. Lee, "An advanced vision-based system for real-time displacement measurement of high-rise buildings," Smart Materials and Structures, vol. 21, no. 12, Article ID 125019, 2012.

[10] Z. C. Luo, Y. Q. Chen, and Y. X. Liu, "Application of GPS in the simulation study of dynamic characteristics of tall buildings," Journal of Wuhan Technical University of Surveying and M Apping, vol. 25, pp. 100-104, 2000.

[11] J. J. Lee and M. Shinozuka, "A vision-based system for remote sensing of bridge displacement," NDT \& E International, vol. 39 , no. 5 , pp. $425-431$.

[12] C. L. Kuang, J. S. Zhang, F. H. Zeng, and W. J. Dai, "Using GPS technology to monitor dynamic response characteristics 
of high-rise building loading by typhoon," Journal of Geodesy and Geodynamics, vol. 32, pp. 139-143, 2012.

[13] T. Luhmann, S. Robson, S. Kyle, and I. Harley, Close Range Photogrammetry, Wiley, Hoboken, NJ, USA, 2007.

[14] R. Jiang, D. V. Jáuregui, and K. R. White, "Close-range photogrammetry applications in bridge measurement: literature review," Measurement, vol. 41, no. 8, pp. 823-834, 2008.

[15] H.-G. Maas, "Photogrammetric techniques for deformation measurements on reservoir walls," in Proceedings of the IAG Symposium on Geodesy for Geotechnical and Structural Engineering, pp. 319-324, Eisenstadt, Austria, 1998.

[16] J. Behmann, A.-K. Mahlein, S. Paulus, H. Kuhlmann, E.-C. Oerke, and L. Plümer, "Calibration of hyperspectral close-range pushbroom cameras for plant phenotyping," ISPRS Journal of Photogrammetry \& Remote Sensing, vol. 106, pp. 172-182, 2015.

[17] B. Caprile and V. Torre, "Using vanishing points for camera calibration," International Journal of Computer Vision, vol. 4, no. 2, pp. 127-139, 1990.

[18] M. Gašparović and D. Gajski, "Two-step camera calibration method developed for micro UAV's," in Proceedings of the ISPRS-International Archives of the Photogrammetry, Remote Sensing and Spatial Information Sciences, vol. XLI-B1, pp. 829-833, 2016.

[19] T. Luhmann, C. Fraser, and H.-G. Maas, "Sensor modelling and camera calibration for close-range photogrammetry," ISPRS Journal of Photogrammetry and Remote Sensing, vol. 115, pp. 37-46, 2016.

[20] G. P. Mateo and J. Luka, "Gimbal influence on the stability of exterior orientation parameters of UAV acquired images," Sensors, vol. 17, no. 2, pp. 401-416, 2017.

[21] X. U. Fang, "The monitor of steel structure bend deformation based on digital photogrammetry," Editoral Board of Geomatics \& Information Science of Wuhan University, vol. 3, pp. 256-260, 2001, in Chinese.

[22] J. I. Jeong, S. Y. Moon, S. G. Choi, and D. H. Rho, "A study on the flexible camera calibration method using a grid type frame with different line widths," in Proceedings of the 41st SICE Annual Conference. SICE 2002, vol. 2, pp. 1319-1324, Osaka, Japan, August 2002.

[23] C. Mingzhi, Y. ChengXin, X. Na, Z. YongQian, and Y. WenShan, "Application study of digital analytical method on deformation monitor of high-rise goods shelf," in Proceedings of the 2008 IEEE International Conference on Automation and Logistics, pp. 2084-2088, Qingdao, China, September 2008.

[24] G. Zhang, G. Guo, C. Yu, and L. Li, "Monitoring dynamic global deflection of a bridge by monocular digital photography," Civil Engineering Journal, vol. 27, no. 2, pp. 168-182, 2018.

[25] G. Zhang, G. Guo, C. Yu, L. Li, S. Hu, and X. Wang, "Monitoring instantaneous dynamic displacements of masonry walls in seismic oscillation outdoors by monocular digital photography," Mathematical Problems in Engineering, vol. 2018, Article ID 4316087, 15 pages, 2018.

[26] G. Zhang, C. Yu, G. Guo et al., "Monitoring sluice health in vibration by monocular digital photography and a measurement robot," KSCE Journal of Civil Engineering, vol. 23, no. 6, pp. 2666-2678, 2019.

[27] G. Zhang, G. Guo, Y. n. Lv, and Y. Gong, "Study on the strata movement rule of the ultrathick and weak cementation overburden in deep mining by similar material simulation: a case study in China," Mathematical Problems in Engineering, vol. 2020, Article ID 7356740, 21 pages, 2020.
[28] G. Zhang, G. Guo, L. Li, and C. Yu, "Study on the dynamic properties of a suspended bridge using monocular digital photography to monitor the bridge dynamic deformation," Journal of Civil Structural Health Monitoring, vol. 8, no. 4, pp. 555-567, 2018. 